\title{
DEGRADACIÓN, REDUCCIÓN Y ELIMINACIÓN DE ÁREAS NATURALES PROTEGIDAS EN EL PERÚ
}

\section{DOWNGRADING, DOWNSIZING AND DEGAZETTING OF PROTECTED AREAS IN PERU}

\author{
Marc J. Dourojeanni ${ }^{1}$
}

\begin{abstract}
Resumen
En un artículo publicado en el 2019 en la revista Science, un grupo de científicos llega a la conclusión que el Perú protagonizó 61 eventos de reducción del nivel de protección y del tamaño, así como de eliminación de áreas protegidas (eventos conocidos en inglés por las siglas PADDD). Eso lo colocaría al país entre los países que peor trata a sus áreas protegidas. Pero, en el Perú solo se tiene conocimiento de un caso. Al revisar la metodología del estudio se confirma que los autores mal interpretaron la información, en especial la referida a los bosques nacionales que en el Perú nunca han sido considerados áreas protegidas. Al parecer, los autores exageraron los argumentos para demostrar la importancia de los PADDD.
\end{abstract}

Palabras clave: áreas naturales protegidas, PADDD, bosques nacionales, legislación.

\begin{abstract}
A paper published in 2019 in the journal Science concludes that Peru starred in 61 events to reduce the level of protection and size as well as to eliminate protected areas (events known by the acronym PADDD). That would place the country among those that most mistreat their protected areas. But, in Peru only one case is known. When reviewing the study methodology, it is confirmed that the authors misinterpreted the information, especially referring to national forests that in Peru have never been considered protected areas. It's seems the authors exaggerated the arguments to demonstrate the significance of PADDD.
\end{abstract}

Key words: natural protected areas, PADDDD, national forests, legislation.

\section{Introducción}

Un reciente artículo, que trata de la problemática del maltrato a las áreas protegidas en el mundo (Golden Kroner et al., 2019) publicado en Science, una de las más prestigiosas revistas científicas del mundo, afirma que el Perú protagonizó 61 eventos de "PADDD", es decir casos de "downgrading" o reducción del nivel de protección, "downsizing" o reducción del tamaño y "degazzeting" o eliminación de sus áreas protegidas (En esta nota se adoptará las siglas PADDD debido a que su uso ya es común en todo el mundo para hacer referencia a degradación, reducción y eliminación de áreas protegidas). Si esa conclusión estuviese acertada implicaría que el Perú, después de Brasil y Colombia sería el país latinoamericano que más perjudicó sus áreas protegidas.

Sin embargo, en el Perú solo se conoce un caso de PADDD, que fue ampliamente controvertido en su oportunidad. Se trata del Parque Nacional Cerros de Amotape (Capital, 2014; SPDA, 2014, 2015) que, como se verá, no implicó un típico caso de PADDD ya que la pequeña área retirada fue compensada con una mayor.

El análisis de la información proporcionada por los autores indica que las justificaciones de las conclusiones respecto al Perú se basan en interpretaciones erradas de textos legales. Este tema ya fue discutido, brevemente, por Dourojeanni (2019). En este artículo se amplían tanto el análisis como los argumentos expuestos.

$\underline{\text { Las afirmaciones del estudio }}$

El artículo de Golden Kroner et al. (2019) recuerda, correctamente, que las áreas protegidas se establecen para salvaguardar la biodiversidad a perpetuidad, pero que la evidencia sugiere que diversos cambios, muchas veces legales, socavan la durabilidad y eficacia de las mismas. Estos cambios; es decir, degradación del nivel de protección, reducciones de tamaño y/o simplemente eliminación (PADDD), ocurren con frecuencia creciente en todos los países del mundo (Mascia \& Pailler, 2011; Mascia et al., 2014), pero en especial en Brasil y en la Amazonía (Bernard et al., 2014; Laue \& Arima, 2016; Pack et al., 2016).

El estudio que se comenta está enteramente basado en información legal. Abarca el nivel mundial, pero trata con mayor énfasis de la situación en los EE UU y en los países amazónicos. Según el artículo, entre 1892 y 2018, 73 países emitieron dispositivos legales que se tradujeron en 3749 eventos PADDD, eliminando la protección de $519857 \mathrm{~km}^{2}$ y reduciendo las regulaciones en $1659972 \mathrm{~km}^{2}$ adicionales. El 78\% de esos eventos se promulgó a partir del año 2000. La mayoría de los eventos de PADDD (62\%) estarían asociados a la extracción de recursos naturales a escala 
industrial, lo que implica que los PADDD pueden comprometer los objetivos de conservación de la biodiversidad. El estudio concluye que se necesitan respuestas de políticas estratégicas para abordar el fenómeno PADDD y mantener áreas protegidas efectivas.

Dentro del universo anterior, el estudio identificó que los gobiernos de los países amazónicos promulgaron 440 eventos PADDD. De esos, el Perú, con 61 eventos PADDD más 3 propuestas de PADDD (Tabla S5 del material suplementario del artículo publicado), sería después de Colombia y Brasil el que más eventos protagonizó en América Latina. Asimismo, el Perú sería el noveno, entre los 78 países del mundo que fueron investigados, que más aplicó PADDD.

En la Tabla 1 se resume lo que Golden Kroner et al. (2019) apuntan sobre el Perú. Esta información no está en el artículo principal, pero sí en el material suplementario provisto por la revista (Tablas S20 y S21). En el artículo hay un mapa de América del Sur donde se ve el Perú con muchos casos PADDD en lugares que, por la reducida escala, no se puede saber a qué corresponden. Según el artículo, los 61 PADDD peruanos fueron realizados entre 1960 y 2017 y afectaron 51 áreas protegidas y a 11826500 hectáreas. De éstas, 42 habrían sufrido degradación del nivel de protección, otras 5 habrían sido afectadas por reducción del tamaño y 14 fueron eliminadas. En la misma Tabla 1 se incluyó lo que esos autores afirman en el caso de la Amazonía peruana donde indican se habría degradado dos áreas, de las que una se redujo, afectando a 2756700 hectáreas. Según los autores, el área total afectada por los eventos PADDD en el Perú "impactó sobre el $23 \%$ del área protegida en el país y que, como resultado de eso, se ha perdido permanentemente el $17 \%$ del área del sistema histórico de áreas protegidas, lo que representa el $4 \%$ de la superficie terrestre total de Perú".

Exceptuando a los bosques nacionales, que son mencionados en bloque, no es posible verificar lo que el artículo informa sobre el Perú, pues ni el texto ni el material que lo sustenta, y que está disponible en Science, se identifican las áreas protegidas a que se refieren, ni tampoco se provee la cita de la referencia bibliográfica que específicamente las menciona. Tampoco se encontró dicha información en el material adicional que los autores del artículo proporcionaron a los que, como el autor de esta nota, procuraron correlacionar los datos con lugares específicos. Apenas existe mención general a "previously unpublished data collected opportunistically" ("datos previos no publicados colectados casuísticamente") y a "meetings with experts" ("reuniones con expertos") y, en anexo, se mencionan, sin mayor precisión, algunas fuentes peruanas como "El Peruano", el Congreso de la República, Sernanp, "Parkswatch" o la Universidad Nacional Agraria de La Molina y, asimismo, dicen haber revisado leyes y documentos ambientales recientes (de 1997 a 2005). Pero no se sabe qué PADDD se atribuye a cada fuente.

Hay otra tabla en la información suplementaria (S22) en la que los autores parecen admitir que algunos de los eventos PADDD para la Amazonía peruana fueron revertidos. No es fácil de entender el cálculo que hacen, pero pareciera que reconocen que 2756700 hectáreas habrían sido mantenidas. Otra vez, no se sabe de qué áreas protegidas están hablando, aunque es probable que en gran parte se trate de la Reserva Nacional Pacaya Samiria, que actualmente posee 2080000 ha.

No se trata en esta nota de analizar o discutir cada detalle de la complicada metodología usada por los autores para ese estudio. Además, eso no es necesario para demostrar que, en el estudio de caso del Perú, ha habido errores importantes.

Análisis de la información

Es bien conocido que en el Perú existió un evento PADDD (Capital, 2014; SPDA, 2014, 2015), este resultó de la aplicación de una ley específica, la Ley 30359 de 2015 (Congreso de la República, 2015), por la que se redujo 277.65 hectáreas al Parque Nacional

Tabla 1. Número y extensión $\left(\mathrm{km}^{2}\right)$ de eventos PADDD en el Perú, y en su Amazonía en particular, de 1961 a 2017.

\begin{tabular}{lccccc}
\hline & Total Eventos PADD & $\mathbf{N}^{\circ}$ ANP afectadas & $\begin{array}{c}\text { Área total } \\
\text { afectada }\end{array}$ & $\begin{array}{c}\text { Área afectada } \\
\text { absoluta }\end{array}$ & Área neta afectada \\
\hline En el Perú en general & & & & & \\
Total PADDD & 61 & $51^{*}$ & $118265(60)$ & $109595(52)$ & $45722(21)$ \\
Degradación & 42 & 34 & $69164(41)$ & $60497(34)$ & $26295(10)$ \\
Reducción & 5 & 5 & $10485(5)$ & $10483(4)$ & $8977(3)$ \\
Eliminación & 14 & 14 & $38616(14)$ & $38616(14)$ & $10449(8)$ \\
En la Amazonía peruana en particular & 3 & & & & \\
Total PADDD & 2 & 3 & $27567(3)$ & $27567(3)$ & 0 \\
Degradación & 1 & 2 & $25469(2)$ & $25469(2)$ & 0 \\
Reducción & 0 & 1 & $2098(1)$ & $2098(1)$ & 0 \\
Eliminación & 0 & 0 & 0 & 0 \\
\hline
\end{tabular}

*Repetición de 10 eventos en las mismas áreas.

Fuente: Referencias al Perú extraídas de tablas S20 y S21 de Supplementary Material de Golden Kroner et al. (2019). 
Cerros de Amotape, las que por el mismo dispositivo legal fueron compensadas con otras 483.87 hectáreas. Además, de no iniciarse la construcción del proyecto de irrigación que justificó el acto hasta 2020, esa área debe revertir al Parque Nacional. Ese acto generó un gran movimiento social en defensa del Parque (SPDA, 2015). Pero, en realidad, hubo otro caso anecdótico de PADDD que Golden Kroner et al. (2019) parecen no haber registrado. En 1965, por error burocrático se creó por ley un minúsculo y técnicamente absurdo "Parque Nacional" Sol y Campo en la periferia de Lima con la única finalidad de brindar un área de picnic en la orilla del río Rímac. Este "parque", que no tuvo área definida y que ni siquiera operó como área recreativa, fue destruido por el río Rímac que trasbordó y limpió el área, situación que fue regularizada por una ley promulgada en la década de 1970 (Dourojeanni, 2018).

Es decir que entre 1960 y 2017, se produjeron solamente dos eventos PADDD en el Perú. Uno ecológicamente insignificante $\mathrm{y}$, el otro, puramente legal. Sin embargo, de acuerdo a Golden Kroner et al. (2019) se produjeron 61 eventos, de los que a este punto del análisis sólo se puede explicar el ya referido caso del Parque Nacional Cerros de Amotape.

Por la metodología usada por Golden Kroner et al. (2019) y, por los mapas en que se señalan los puntos en los que ellos deciden hubo eventos PADDD, resulta probable que en el Perú hayan considerado cuatro situaciones: (i) mediciones nuevas y ajustes de tamaños o modificaciones de límites de áreas protegidas existentes, (ii) ampliaciones y fusiones de áreas protegidas; (iii) extinción de bosques nacionales y; (iv) otros eventos diversos, aparentemente en Costa y Sierra, que no se consiguió identificar.

LA FLEXIBILIDAD DE LOS LÍMITES. Se debe tener en cuenta que el primer parque nacional peruano fue establecido en 1961. Antes, con la relativa excepción de las islas guaneras, cuya finalidad era la producción de fertilizante, no existía nada en la legislación peruana, o en la práctica, que pudiera ser equiparado a un área natural protegida (Dourojeanni, 1968, 1976, 2009; Dourojeanni \& Ponce, 1978). La primera mención legal al tema de áreas protegidas apareció con la creación del Parque Nacional Cutervo (1961) y fue ratificada con la Ley Forestal 14552 de 1963 (Perú, 1963) que reconoció los parques nacionales como la única categoría de área protegida peruana. De otra parte, los tres primeros parques nacionales (Cutervo, Tingo María y Sol y Campo) fueron creados sin indicación de su superficie ni de sus límites. Era, pues inevitable que en años subsiguientes esos límites se establecieran. Todo indica que esas acciones fueran incluidas como eventos PADDD ya que, obviamente, debieron ser sancionados por las leyes.

De otra parte, las áreas protegidas establecidas entre 1965 y comienzo de los años 1990 eran diseñadas en base a información cartográfica convencional, usando metodologías geográficas -por ejemplo, fotografías aéreas- y topográficas de la época, es decir con muchas imprecisiones que años más tarde necesitaron correcciones. Las revisiones con instrumentos modernos, como las de posicionamiento geográfico global (GPS), obligaron a varios pequeños ajustes territoriales que, en general, implicaron modificaciones legales (decretos supremos). Podían o no motivar pequeños cambios en la superficie de las áreas, para más o para menos. Una interpretación puramente legal de esos ajustes, como parece haber sido el caso, podrían ser interpretadas como casos de PADDD.

FUSIONES DE ÁREAS PROTEGIDAS. Es evidente que Golden Kroner et al. (2019) incluyeron como PADDD varios eventos de fusión o modificación de categoría de áreas protegidas. Por ejemplo, como anticipado, parece que consideraron PADDD el caso de la Reserva Nacional Pacaya y Samiria. Esta fue resultado de la fusión de otras dos áreas creadas inicialmente en 1968 como reserva nacional y coto de caza, respectivamente. En 1972 ambas se juntaron en la actual Reserva Nacional, sin reducción del tamaño. Tal parece que, para Golden Kroner et al. (2019), esos actos implicaron "eventos PADDD". Lo mismo ocurrió cuando el Santuario Nacional Pampas del Heath, creado en 1983, fue incorporado en 1996 dentro del nuevo Parque Nacional Bahuaja-Sonene, sin perder terreno, pero contabilizaron un PADDD de eliminación de área protegida. Y pueden citarse otros casos similares. También debieron incluir el caso del Parque Nacional del Manu, que se creó sobre el antiguo Bosque Nacional del Manu y que además fue ampliado hasta en dos ocasiones y, asimismo, los casos de otros bosques nacionales total o parcialmente transformados en áreas protegidas (Tabla 2), como el Bosque de Protección Biavo, el Santuario Nacional Tabaconas-Namballe o los parques nacionales Abiseo, Yanachaga-Chemillén, Cordillera Azul, Otishi y la Reserva Nacional de Tumbes.

En resumen, aunque sancionados por dispositivos legales, ninguno de los casos citados califica como PADDD. Al contrario, en todos esos casos primó el interés de conservar más y mejores muestras de los ecosistemas naturales.

EL CASO DE LOS BOSQUES NACIONALES. El grueso de los PADDD incluidos por Golden Kroner et al. (2019) parece corresponder al caso de los bosques nacionales. Afirman, en sus anexos, que 13 PADDD ocurrieron en 1996, cuando "se retiraron los bosques nacionales del sistema de áreas protegidas para dedicarlas a la explotación comercial de madera". Pero los bosques nacionales no eran áreas protegidas y siempre estuvieron dedicados al manejo forestal con fines de producción de madera (Perú, 1963, 1975; Dourojeanni, 2009).

Para que exista un evento PADDD es condición sine qua non que exista un área natural protegida. Si no existe un área protegida, legalmente o en la práctica, no cabe haber PADDD. Si en el Perú los 
bosques nacionales, desde su concepción, estuvieron destinados a la producción maderera, no es razonable incluirlos como áreas protegidas. Los bosques nacionales fueron creados entre 1956 y 1973, aunque la mayoría datan de 1963. Esos bosques, en realidad fueron 18 a lo largo del tiempo (Tabla 2), sumaban 7826000 hectáreas a mediados de los años 1970. Si se suma a esa superficie el caso ya comentado de la Reserva Nacional Pacaya Samiria (2080000 ha), queda explicada en un $84 \%$ la cifra citada por Golden Kroner et al. (2019), que menciona PADDD afectando 11826500 hectáreas.

El artículo 15 de la primera Ley Forestal del Perú, Ley 14552 de 11 de julio de 1963 (Perú, 1963), declara: "Bosques Nacionales, son establecidos con carácter definitivo, principalmente, con fines de protección o producción permanente de madera y productos forestales secundarios y tienen el carácter de inalienables". El artículo 16 de la misma ley se refiere a los parques nacionales y dice: "son establecidos con carácter definitivo para ser destinados a la protección y conservación de las bellezas escénicas naturales de la flora y la vida silvestre de importancia nacional, para ser puestos al servicio público, quedando reglamentada la explotación agropecuaria forestal, la caza y la pesca". Es decir, aunque el término "protección” en el artículo 15 podría ser considerado como una mención a la protección de la biodiversidad, interpretación errónea también atribuida por Espichan (2012), es evidente que, en realidad, se refiere a la protección del bosque para su uso futuro y que los bosques nacionales son para la producción, mientras que los parques nacionales eran, ellos sí, para la conservación de la naturaleza. La legislación forestal de 1975, que con relación a bosques nacionales Espichan evita mencionar, aclara perfectamente la diferencia.

El artículo 11 del capítulo "De los Bosques" de la Ley Forestal y de Fauna Silvestre 21147, de 13 de mayo de 1975 (Perú, 1975), es aún más contundente: "Se denomina Bosques Nacionales, los bosques naturales declarados aptos para la producción permanente de madera, otros productos forestales y de fauna silvestre, cuya utilización solo podrá ser realizada directa y exclusivamente por el Estado. La declaración se hará por Resolución Suprema". En ninguna parte de ese texto se hace referencia a protección de la naturaleza o a conservación de la biodiversidad, que es el argumento usado por Golden Kroner et al. (2019) para incluirlos en su cálculo. Claramente se refiere exclusivamente al uso de la madera y otros productos. Marcando la diferencia, en esa ley misma existe otro capítulo que se refiere a las áreas protegidas o unidades de

Tabla 2. Bosques Nacionales posiblemente incluidos en el estimado de PADDD en el Perú de Golden Kroner et al. (2019).

\begin{tabular}{|c|c|c|c|c|c|}
\hline \multicolumn{2}{|c|}{ RP y BN posiblemente incluidos } & \multirow{2}{*}{$\frac{\text { Área }\left(\mathbf{k m}^{\mathbf{2}}\right)}{721}$} & \multirow{2}{*}{$\begin{array}{c}\text { Creación } \\
1957\end{array}$} & \multirow{2}{*}{$\begin{array}{c}\text { Eliminación } \\
*\end{array}$} & \multirow{2}{*}{\begin{tabular}{l}
\multicolumn{1}{c}{$\begin{array}{c}\text { Áreas protegidas } \\
\text { resultantes }\end{array}$} \\
PN Cerros de Amotape \\
RN Tumbes
\end{tabular}} \\
\hline 1 & BN Tumbes & & & & \\
\hline 2 & BN Mesapata & 261 & 1956 & 1975 & - \\
\hline 3 & $\begin{array}{l}\text { BN Morona-Pastaza- } \\
\text { Marañón }\end{array}$ & 3750 & 1963 & $*$ & Sitio RAMSAR \\
\hline 4 & BN Biavo-Cordillera Azul & 20845 & 1963 & $*$ & PN Cordillera Azul \\
\hline 5 & BN Mariscal Cáceres & 3370 & 1963 & & - \\
\hline 6 & BN Apurimac & 20710 & 1963 & $*$ & PN Otishi \\
\hline 7 & BN Manu & 3000 & 1963 & $*$ & PN Manu \\
\hline 8 & BN Oso Perdido & 29 & 1963 & $*$ & $\begin{array}{l}\text { SN Tabaconas } \\
\text { Namballe }\end{array}$ \\
\hline 9 & BN Kcosñipata & 3118 & 1963 & 1975 & - \\
\hline 10 & BN Huambramayo & 3700 & 1963 & 1975 & - \\
\hline 11 & BN Oxapampa & 3000 & 1963 & $*$ & PN Yanachaga \\
\hline 12 & BN Semuya-San Gabán & 64 & 1963 & 1975 & - \\
\hline 13 & BN Alto Mayo & 3100 & 1963 & $*$ & BP Alto Mayo \\
\hline 14 & BN Tahuayo & 1058 & 1963 & 1975 & - \\
\hline 15 & BN Nanay & 1200 & 1963 & 1975 & - \\
\hline 16 & BN Alexander von Humboldt & 6450 & 1965 & $*$ & Estación Experimental \\
\hline 17 & BN Iparía & 2550 & 1966 & 1977 & - \\
\hline \multirow[t]{2}{*}{18} & BN San Ignacio & 1800 & 1973 & $*$ & $\begin{array}{l}\text { SN Tabaconas } \\
\text { Namballe }\end{array}$ \\
\hline & TOTAL & 78726 & & & \\
\hline
\end{tabular}

BN: Bosque Nacional, PN: Parque Nacional, SN: Santuario Nacional, RN: Reserva Nacional.

* Estos bosques fueron integralmente transformados en áreas protegidas y otros usos.

Fuentes : Malleux (1975), Dourojeanni (2009), SERNANP (2019). 
conservación y que incluye parques nacionales, reservas nacionales, santuarios nacionales y santuarios históricos, en los cuales sí se menciona la conservación de la naturaleza.

Los bosques nacionales tampoco eran internacionalmente considerados como áreas protegidas hasta fines de la década de 1970 y comienzo de 1980, lapso en el que en su mayoría existieron en el Perú. La Convención para la Protección de la Flora y Fauna y las Bellezas Escénicas de América (Convención Washington), del 12 de octubre de 1940 (OEA, 1940), que ha sido la base del comportamiento peruano en relación con la conservación de la naturaleza antes de la Ley Forestal de 1963 (Perú, 1963), solo menciona parques nacionales, reservas nacionales, monumentos naturales y reservas de regiones vírgenes. Ninguna de esas categorías corresponde a la de bosques nacionales que ya existían desde mucho antes en los EE UU. De otra parte, los bosques nacionales no fueron considerados áreas protegidas antes de la reforma de las categorías adoptadas por la IUCN (1978), es decir, después de que la mayoría de los bosques nacionales peruanos ya habían sido extinguidos (Tabla 2) y, especialmente, después del Congreso Mundial de Parques Nacionales de 1982, en Bali, Indonesia, donde se decidió dar impulso a la categoría VIII -áreas de uso múltiple o de manejo de recursos- que puede, pero no determina, incluir bosques nacionales (McNeely \& Miller, 1983). Ni siquiera figuraban para los EEUU en la Lista de las NN UU de Parques Nacionales y Reservas Equivalentes de 1975 (IUCN, 1975) y recién aparecen, pero como categoría III -monumentos naturales- en la Lista de 1990 (IUCN, 1990), siempre y cuando sean así propuestos por la autoridad nacional. Pero los bosques nacionales peruanos jamás fueron mencionados en una lista de la IUCN, por la simple razón de que no fueron establecidos para fines de conservación y sí para producción de madera y porque el Perú jamás propuso incorporarlos a las listas de la IUCN áreas protegidas. De cualquier modo, como se sabe, el término "bosque nacional" fue extinguido de facto por derogación de la Ley 21147 (Perú, 1975), mediante la Ley Forestal 27308 de 15 de julio de 2000 (Perú, 2000) que la sustituyó.

Sin embargo, entre 1990 y 1996 ocurrió un hecho sui generis que podría justificar el "error" de la interpretación de Golden Kroner et al. (2019), que ya había sido cometido por Espichan (2012) quien, en un análisis superficial y puramente legal del tema, concluyó que el Decreto Supremo 010-90-AG que aprobó la versión 1990 del Sistema Nacional de Áreas Naturales Protegidas (SINANPE), había incluido los bosques nacionales (MINAGRI, 1990). Este decreto fue un error detectado de inmediato, pero que fue formalmente corregido solo cinco años más tarde, por el Decreto Supremo 011-96-AG (MINAGRI, 1996). A pesar del Decreto Supremo 10-90-AG los bosques nacionales nunca pasaron al SINANPE ni fueron administrados por la entonces Dirección General de Áreas Protegidas del INRENA. Una prueba adicional de esto es que ninguno de los varios compendios legales sobre áreas protegidas (Andaluz \& Valdez, 1987; SPDA \& INRENA, 2002; MINAM, 2005; Solano, 2009) menciona a los bosques nacionales como áreas protegidas.

También llama la atención que, a pesar del énfasis legalista, no se ha llevado en cuenta el nivel jerárquico de los dispositivos legales. En el caso de los bosques nacionales, que parecen haber sido el "plato principal" del ejercicio, tanto Espichan (2012) como Golden Kroner et al. (2019) han omitido el hecho de que su inclusión errónea como áreas protegidas fue realizada mediante un decreto supremo cuya finalidad, además, no era definir o caracterizas áreas protegidas sino, simplemente, aprobar una versión del sistema nacional de áreas protegidas. La definición de bosque nacional estaba claramente establecida por la Ley 21147 (Perú, 1975), que no fue modificada por el tal decreto. Es decir, que la mención de los bosques nacionales en ese decreto no los convertía en áreas protegidas.

OTROS CASOS. De otra parte, tratando de entender los puntos PADDD que figuran en el mapa provisto por Golden Kroner et al. (2019), se ha especulado que algunos se refieran a áreas municipales. Pero, aunque la legislación peruana sobre áreas protegidas no prevé áreas municipales (Solano, 2009), el Decreto Supremo 010-99-AG, que aprobó la versión 1999 del plan director de áreas naturales protegidas (MINAGRI, 1999), mencionó la posibilidad de establecerlas y, por eso, es probable que algunas fueran establecidas por autoridades locales. Pero éstas nunca fueron reconocidas oficialmente, por lo tanto, no pueden configurar procesos PADDD. Si ese fuera el caso, se trata de otro error ya que, además, tales lugares siempre han sido urbanos o periurbanos, para recreación y nunca dedicados a conservar la biodiversidad.

Con base a los criterios usados por Golden Kroner et al. (2019) hasta es posible que incluyeran las islas guaneras, antes bajo jurisdicción del Ministerio de Agricultura que, en 2009, pasaron a ser un área protegida. Finalmente, los autores indican no haber incluido como PADDD las zonas reservadas, por ser transitorias, pero todo indica que si incluyeron por lo menos cinco de ellas: Aymara-Lupaka, Humedales de Puerto Viejo, Ancón, Algarrobal El Moro y Puerto Viejo.

\section{Discusión}

Un primer aspecto en el que se debe insistir es que el estudio de Golden Kroner et al. (2019) es estrictamente legalista. No se ha interesado en conocer la realidad en el terreno ni en comprender el motivo que originó los cambios legales. Simplemente partió de la idea de que todo cambio registrado legalmente era un PADDD. Sin embargo, todos los casos de PADDD 
usados, excepto el ya citado del Parque Nacional Cerros de Amotape, han tenido como finalidad precipua favorecer las áreas protegidas. No obstante, esas mejoras han sido presentadas como hechos mal intencionados negativos para la tarea de conservar la naturaleza.

PADDD es un término con connotación única y absolutamente negativa para la conservación de la biodiversidad. No existe "PADDD bueno". Entonces, no se comprende el motivo de aplicarlo a eventos claramente favorables a la conservación de la diversidad biológica, como en el caso de ampliaciones, correcciones o fusiones de áreas o aumento del nivel de protección. Es obvio que los autores descartaron cualquier material que no apoyaba sus intenciones de mostrar un escenario mucho peor que la realidad. El trato dado por los autores, al componente peruano en su estudio, indica que ellos han buscado exagerar el resultado, haciendo parecer como perjudicial a la conservación actos que bajo cualquier criterio la favorecieron.

Desde la década de 1960 existen numerosos trabajos publicados sobre las áreas naturales protegidas del Perú que explican, para diferentes periodos de la historia, los avances y retrocesos en el establecimiento y defensa de esas áreas (Dourojeanni, 1968, 1976, 2009, 2018; Vílchez, 1968; Dourojeanni \& Ponce, 1978, Dourojeanni \& Ríos, 1983; Solano, 2005, 2009, 2020). Estas referencias, entre decenas más detalladas y referidas a áreas protegidas específicas, hubieran aclarado las dudas de los autores extranjeros y evitado sus errores. En ellas se revela claramente que hubo diversas y hasta graves iniciativas contra las áreas protegidas del país, lo que hubiera podido significar PADDD, siendo los casos del Manu, y el de Paracas, algunos de los más conocidos. Pero también se explica cómo esos atentados fueron todos evitados. Sin embargo, ninguna de esas fuentes de información fue consultada por los autores, que únicamente usaron las que parecían más convenientes a sus propios propósitos.

\section{Conclusiones}

No hay duda que los verdaderos eventos PADDD son un problema creciente para la conservación de la diversidad biológica y que son cada vez más graves y frecuentes. Es importante cuantificar esos hechos y denunciarlos. Lo que no es fácil entender es el propósito de exagerarlos y, en el caso analizado, prácticamente de inventar casos de PADDD donde evidentemente no los hay. El manipuleo tan extremo e imprudente de los datos peruanos lesiona el bien ganado prestigio del Perú en el tema de las áreas naturales protegidas y habla muy mal de la seriedad de una revista prestigiosa como Science (por las comunicaciones personales del autor de esta nota con la autora principal del artículo, meses antes de la publicación, se sabe que ella conocía los hechos detallados en esta nota, los que descartó. Y también es conocido que varios otros expertos peruanos insistieron con ella en que su interpretación de PADDD estaba equivocada).

Y eso deja serias dudas sobre lo que los autores denuncian de otros países. Lo que hicieron no es ciencia, es demagogia, y en lugar de ayudar a la causa ambiental la perjudica, restando credibilidad a la lucha por conservar la diversidad biológica y, por cierto, es un serio desestimulo para los que en el terreno luchan por salvar algo de la naturaleza.

\section{Literatura citada}

Andaluz A. \& Valdez W. 1987. Derecho Ecológico Peruano. Inventario Normativo. Gredes, Lima.

Bernard E., Penna L.A.O. \& Araújo E. 2014. Downgrading, downsizing, degazettement, and reclassification of Protected Areas in Brazil. Conservation Biology, 28(4): 939-950. https://doi.org/10.1111/cobi.12298.

Capital. 2014. Advierten riesgo de recorte del Parque Nacional Cerros de Amotape. Capital, 7 de noviembre 11:54 AM. https://capital.pe/actualidad/advierten-riesgode-recorte-del-parque-nacional-cerros-de-amotapenoticia-745849.

Congreso de la República. 2015. Ley No 30359: Ley para la implementación de acuerdos binacionales entre Perú y Ecuador y ejecución del Proyecto Especial Binacional Puyango-Tumbes. Diario El Peruano (Normas Legales), jueves 12 de noviembre, Año XXXII(13463): 566154566160.

https://busquedas.elperuano.pe/download/url/ley-parala-implementacion-de-acuerdos-binacionales-entre-peley-n-30359-1311209-1.

Dourojeanni M.J. 1968. Estado actual de la conservación de la flora y de la fauna en el Perú. Washington, DC. Ciencia Interamericana, 9(1-6): 1-12.

Dourojeanni M.J. 1976. Machu Picchu and Peru's national system of conservation units. Washington D.C. Parks, 1(2): 8-11.

Dourojeanni M.J. 2009. Crónica Forestal del Perú. Univers. Nac. Agraria La Molina. Editorial San Marcos, Lima. .

Dourojeanni M.J. 2018. Áreas Naturales Protegidas del Perú: El Comienzo. Univ. Nac. Educ. Guzmán y Valle. Grijley, Lima. https://www.academia.edu/37923859/\%C3\%81REAS_ NATURALES_PROTEGIDAS_DEL_PER\%C3\%9A_E L_COMIENZO.

Dourojeanni M.J. 2019. Perú: ¿Uno de los tres países de América Latina que peor trata sus áreas protegidas? Actualidad Ambiental. SPDA. Lima Miércoles 12 de junio, 2019. https://www.actualidadambiental.pe/peruuno-de-los-tres-paises-de-america-latina-que-peor-tratasus-areas-protegidas/.

Dourojeanni M.J. \& Ponce C.F. 1978. Los Parques Nacionales del Perú. Instituto de la Caza Fotográfica (INCAFO). Colección "La Naturaleza en Iberoamérica". Madrid.

Dourojeanni M.J. \& Ríos M. 1983. Un enfoque crítico sobre el Sistema Nacional de Unidades de Conservación del Perú. Revista Forestal del Perú, 12(1): 12-22. http://cedinfor.lamolina.edu.pe/Articulos_RFP/Vol11_n o1-2_82-83_(15)/vol11_art12.pdf. 
Espichan E.M.A. 2012. La conservación de las áreas naturales protegidas y los fenómenos PADDD (degradación, reducción y degazzettement de áreas naturales protegidas). Lima. Desde el Sur, 5(1): 35-47. https://revistas.cientifica.edu.pe/index.php/desdeelsur/art icle/view/147.

Golden Kroner R.E., Qin S., Cook C.N., Krithivasan R., Pack S.M., Bonilla O.D., Cort-Kansinally K.A., Coutinho B., Feng M., Martínez M.I., He Y., Kennedy Ch.J., Lebreton C., Ledezma J.C., Lovejoy Th.E., Luther D.A., Parmanand Y., Ruíz-Agudelo C.A., Yerena E., Morón V. \& Mascia M.B. 2019. The uncertain future of protected lands and waters. Science, 364(6443): 881-886. DOI: $10.1126 /$ science.aau5525.

IUCN. 1975. United Nations List of National Parks and Equivalent Reserves. IUCN, Morges. https://portals.iucn.org/library/sites/library/files/docume nts/NS-033.pdf.

IUCN. 1978. Categories, objectives and criteria for protected areas. Committee on Criteria and Nomenclature. Commission on National Parks and Protected Areas. IUCN. Morges, Switzerland https://portals.iucn.org/library/sites/library/files/docume nts/1978-003.pdf.

IUCN. 1990. United Nations List of National Parks and Protected Areas. IUCN, Gland, Switzerland and Cambridge,

UK. https://portals.iucn.org/library/sites/library/files/doc uments/UNLNP-1990-001.pdf.

Laue J. \& Arima E. 2016. What Drives Downsizing of Protected Areas? A Case Study of Amazon National Park. Journal of Latin American Geography, 15(2): 7-31. DOI: 10.1353/lag.2016.0013.

Malleux J. 1975. Mapa Forestal del Perú. Memoria Explicativa. Univ. Nac. Agraria La Molina. Lima.

Mascia M.B. \& Pailler S. 2011. Protected area downgrading, downsizing, and degazettement (PADDD) and its conservation implications. Conservation Letters, 4(1): 920. DOI: 10.1111/j.1755-263x.2010.00147.x.

Mascia M.B., Pailler S., Krithivasan R., Roshchanka V., Burns D., Mlotha M.J., Murray D.R. \& Peng N. 2014. Protected area downgrading, downsizing, and degazettement (PADDD) in Africa, Asia, and Latin America and the Caribbean, 1900-2010. Biological Conservation, 169: 355-361.

https://europepmc.org/article/agr/ind605360757.

McNeely J.A. \& Miller K.R. 1983. IUCN, National Parks, and Protected Areas: Priorities for Action Executive. Environmental Conservation, 10(1): 13-20. https://doi.org/10.1017/S0376892900011826. https://core.ac.uk/download/pdf/85222674.pdf.

MINAGRI. 1990. Decreto Supremo No 010-90-AG: Sistema Nacional de Unidades de Conservación. (Diario Oficial) El Peruano (Normas Legales), Lima, sábado 24 de marzo de 1990: 83030-83032.

http://extwprlegs1.fao.org/docs/pdf/per11490.pdf.

MINAGRI. 1996. Decreto Supremo No 011-96-AG: Determinan zonas de protección ecológica de la región de selva. (Diario Oficial) El Peruano (Normas Legales). http://extwprlegs1.fao.org/docs/pdf/per11492.pdf.
MINAGRI. 1999. Decreto Supremo No 010-99-AG: Aprueban el Plan Director de las Areas naturales Protegidas. (Diario Oficial) El Peruano (Normas Legales), Año XVII(6865): 171941-171962. http://biblioteca.unmsm.edu.pe/redlieds/Recursos/archiv os/Legislacion/Peru/DS010-99-AG.pdf.

MINAM. 2005. Compendio de la Legislación Ambiental Peruana. Volumen IX, Áreas Naturales Protegidas. Documento Preliminar. Lima. http://infobosques.com/portal/wpcontent/uploads/2016/05/Compendiolegislacion09.pdf.

OEA (Organización de los Estados Americanos). 1949. Convención para la Protección de la Flora y Fauna y las Bellezas Escénicas de América (Convención Washington). 12 de octubre de 1940. https://www.oas.org/juridico/spanish/tratados/c-8.html. https://ecohis.jmarcano.com/areasprotegidas/antecedentes/washington/convencion/.

Pack S.M., Ferreira M.N., Krithivasan R., Murrow J., Bernard E. \& Mascia M.B. 2016. Protected area downgrading, downsizing, and degazettement (PADDD) in the Amazon. Biological Conservation, 197: 32-39. DOI: 10.1016/j.biocon.2016.02.004.

Perú. 1963. Ley Forestal y de Caza No 14552. https://leyes.congreso.gob.pe/Documentos/Leyes/14552. pdf. http://extwprlegs1.fao.org/docs/pdf/per93771.pdf. https://www.ecolex.org/details/legislation/decreto-leyno-14552-crea-el-servicio-forestal-y-de-caza-lexfaoc093771/.

Perú. 1975. Ley Forestal y de Fauna Silvestre No 21147. https://leyes.congreso.gob.pe/Documentos/Leyes/21147. pdf. http://www.legislacionforestal.org/wpcontent/uploads/2010/08/dl-21147.pdf.

Perú. 2000. Ley No 27308: Ley Forestal y de Fauna Silvestre. (Diario Oficial) El Peruano (Normas Legales), XVIII(7328): 190283-190289.

https://leyes.congreso.gob.pe/Documentos/Leyes/27308. pdf.

https://www.serfor.gob.pe/pdf/normatividad/2000/ley/L EY\%2027308.pdf.

SERNANP. 2019. Nuestras áreas naturales protegidas. https://www.sernanp.gob.pe/nuestras-areas-naturalesprotegidas.

Solano P. 2005. La Esperanza es Verde. SPDA, Lima. https://spda.org.pe/wpfb-file/20060829111038_-pdf.

Solano P. 2009. Marcos Regulatorios Nacionales de Áreas Protegidas: Perú. IUCN-EPLP No. 81. https://www.iucn.org/downloads/peru_es.pdf.

Solano P. 2020. ¿Qué es un Parque Nacional? El ABC de las áreas naturales protegidas en el Perú. Ed. P. Solano. Lima. https://biblioteca.spda.org.pe/biblioteca/catalogo/_data/2 0200921224139_Que\%20es\%20un\%20parque\%20nacio nal.pdf.

SPDA \& INRENA. 2002. Compendio de Legislación de Áreas Naturales Protegidas. Sociedad Peruana de Derecho Ambiental e INRENA. Lima, Perú. https://spda.org.pe/wpfb-file/libro-final-pdf/.

SPDA. 2014. Tumbes: Gobierno anunció que por primera vez recortarán un Parque Nacional para proyecto de irrigación. Actualidad Ambiental, viernes 7 de Noviembre.

https://www.actualidadambiental.pe/tumbes-gobiernoanuncio-recorte-de-parque-nacional-cerros-de-amotapepara-proyecto-de-irrigacion/. 
DEGRADACIÓN, REDUCCIÓN Y ELIMINACIÓN DE ÁREAS NATURALES PROTEGIDAS EN EL PERÚ Enero - Julio 2021

SPDA. 2015. Comisión Agraria aprobó dictamen sobre redefinición del Parque Nacional Cerros de Amotape. Actualidad Ambiental. Martes 2 de Junio. https://www.actualidadambiental.pe/?p=30474.
Vílchez S. 1968. Parques Nacionales del Perú. Ed. La Promotora. Lima.

${ }^{1}$ Profesor emérito. Universidad Nacional Agraria La Molina. Lima / Perú. marc.dourojeanni@ gmail.com. 